\title{
A systematic review and meta-analysis of the clinical efficacy of anterior lumbar interbody fusion in the treatment of orthopedic spondylolisthesis
}

\author{
Xiyan $\mathrm{Xu}^{1}$, Xiuyang $\mathrm{Li}^{2}$, Tao Yang ${ }^{3}$ \\ ${ }^{1}$ Department of Orthopedic Surgery, Fifth People's Hospital of Chongqing, University of Chinese Academy of Sciences Chongqing Renji Hospital, \\ Chongqing, China; ${ }^{2}$ Department of Orthopedics, Seventh People's Hospital of Chongqing, Chongqing, China; ${ }^{3}$ Chongqing Municipality Clinical \\ Research Center for Geriatric Diseases, Chongqing University Three Gorges Hospital, Chongqing, China \\ Contributions: (I) Conception and design: X Xu, T Yang; (II) Administrative support: X Li; (III) Provision of study materials or patients: X Xu, T \\ Yang; (IV) Collection and assembly of data: All authors; (V) Data analysis and interpretation: All authors; (VI) Manuscript writing: All authors; (VII) \\ Final approval of manuscript: All authors. \\ Correspondence to: Tao Yang. 165 Xincheng Road, Wanzhou District, Chongqing, China. Email: yangtdoc@163.com.
}

Background Many studies have reported the advantages of anterior lumbar interbody fusion (ALIF), but the technique is associated with many complications involving nerve injury. This meta-analysis compared the outcome indicators associated with 2 fusion methods, namely, ALIF and posterolateral fusion (PLF). The clinical efficacy of ALIF was explored to provide evidence-based data for the determination of surgical methods for treating orthopedic spondylolisthesis.

Methods: Relevant literatures were retrieved from the CBMdisc, CNKI, PubMed, EBSCO, MEDLINE, Science Direct, and Cochrane databases. Keywords in Chinese and English included spondylolisthesis, spine, surgical treatment, ALIF, and PLF. Data including the visual analogue scale (VAS) score, the Oswestry Disability Index (ODI), time of operation, and fusion rate were collated. According to Cochrane manual, Rev Man 5.3 software was used for analysis.

Results: A total of 6 articles were included in this meta-analysis. There were significant differences in intraoperative blood loss $[Z=3.34$; mean difference $(M D)=-142.54 ; 95 \%$ confidence interval $(C I):-226.17$ to $-58.92 ; \mathrm{P}=0.0008]$ and operation time $(\mathrm{Z}=5.45 ; \mathrm{MD}=-54.31 ; 95 \% \mathrm{CI}$ : -73.83 to $-34.79 ; \mathrm{P}<0.00001)$ between patients in the ALIF group and patients in the PLF group. Significant differences were observed in VAS score $(\mathrm{Z}=3.55 ; \mathrm{MD}=-1.04 ; 95 \% \mathrm{CI}:-1.62$ to $-0.47 ; \mathrm{P}=0.0004)$ nor ODI score $(\mathrm{Z}=3.07 ; \mathrm{MD}=-6.33$; 95\% CI: -10.37 to $-2.28 ; \mathrm{P}=0.002)$ between the ALIF group and the PLF group. Interestingly, there was a significant difference in the hospitalization time between the 2 groups $(\mathrm{Z}=2.39 ; \mathrm{MD}=-1.48 ; 95 \% \mathrm{CI}:-2.70$ to $-0.27 ; \mathrm{P}=0.02$ ). Bone fusion rate was no significantly different between patients in the ALIF group and patients in the PLF group [ $\mathrm{Z}=0.43$; odds ratio $(\mathrm{OR})=0.42 ; 95 \% \mathrm{CI}: 0.01$ to $21.82 ; \mathrm{P}=0.66]$.

Discussion: The results of this meta-analysis confirmed that ALIF can effectively improve the degree of spondylolisthesis, provide superior structural stability, and ensure surgical efficacy.

Keywords: Anterior lumbar interbody fusion (ALIF); orthopedic; spondylolisthesis; clinical efficacy; meta-analysis

Submitted Oct 27, 2021. Accepted for publication Dec 08, 2021.

doi: 10.21037/apm-21-3330

View this article at: https://dx.doi.org/10.21037/apm-21-3330

(c) Annals of Palliative Medicine. All rights reserved. 


\section{Introduction}

Spondylolisthesis refers to the pathological process characterized by the forward or backward displacement of the lower vertebral body (1). The condition occurs in the posterior tethered structure, where the position of vertebral arch collapses, steps and setback surfaces appear, and the spine leaves the normal position with fore-and-after displacement. Spondylolisthesis is divided into 5 degrees. A degree I slippage is between $1-24 \%$ of the anterior and posterior diameter of the lower vertebral body, degree II is between $5-49 \%$, degree III is between $50-74 \%$, and degree IV is $75-99 \%$. Complete displacement is classified as degree V where slippage exceeds $100 \%$. Degrees I and II are defined as mild spondylolisthesis, degrees III and IV are severe spondylolisthesis, and degree $\mathrm{V}$ is lumbar prolapse $(2-4)$. At present, the recommended treatment for severe spondylolisthesis is $360^{\circ}$ perivertebral fusion surgery, which greatly increases the bone graft fusion rate and reduces the failure rate of the operation.

Anterior lumbar interbody fusion (ALIF) was first proposed and applied clinically by Burns in the 1990s. Compared with posterior lumbar interbody fusion, ALIF can completely remove the diseased intervertebral disc, place a larger interbody fusion cage, increase the bone grafting area and improve the fusion rate. In terms of biology, it is beneficial to restore lordosis, keep intact posterior structure and enhance the stability of spine (5). On the premise of not destroying the lumbar posterior lamina, protect the integrity of the lumbar posterior tension structure; ALIF does not interfere with the nerve structure in spinal canal, and can reduce bleeding and the incidence of complications. In addition, patients recover quickly after operation, and they can get out of bed in advance to improve their quality of life (6-8). However, ALIF can not directly decompress the nerve root, but indirectly decompress the nerve root by restoring the intervertebral height. The operation is difficult, the technical requirements are high, and it is easy to hurt important blood vessels and nerves in pelvic area and lower abdominal nerve (9).

Theoretically, ALIF has advantages in clinical effect, reconstruction of spinal stability, correction of lordosis angle, restoration of dislocation balance, and fusion rate of bone graft, etc. However, at present, there is no systematic evaluation of the clinical effect of ALIF in treating spondylolisthesis in China for clinical reference. The innovation of this study lies in using the method of meta-analysis, and comparing the outcome indexes of ALIF and posterolateral fusion (PLF), in order to explore the clinical efficacy of ALIF and provide evidence-based basis for the determination of surgical methods of orthopedic spondylolisthesis. We present the following article in accordance with the PRISMA reporting checklist (available at https://dx.doi.org/10.21037/apm-21-3330).

\section{Methods}

\section{Literature search}

The CBMdisc, CNKI, PubMed, EBSCO, MEDLINE, Science Direct, and Cochrane databases were search for relevant literatures relating to randomized controlled trials (RCTs) published from the establishment of the database to June 20, 2021.

The object search words for both English and Chinese databases were spondylolisthesis, spine, surgical treatment, ALIF, PLF. The keywords related to the observation indexes were visual analysis scale score, Oswestry Disability Index (ODI), time of operation, and fusion rate. A combination of subject words and free words was used for multiple searches to obtain references that can be included, and the search engine was used to identify each literature. Relevant experts and researchers in the field were contacted to obtain data regarding the latest research progress. The Rev Man 5.3 software provided by the Cochrane Collaboration Network was used to evaluate the quality of the literature.

\section{Literature inclusion and exclusion criteria}

Studies which were prospective RCTs involving orthopedic spondylolisthesis patients undergoing ALIF or PLF were included.

Individual cases, non-research literature, non-RCTs, duplicate publications, and papers with missing data or where there was no original data, were excluded from this meta-analysis.

\section{Clinical efficacy evaluation index}

The clinical evaluation indexes including the visual analogue scale (VAS), the ODI, and treatment score, the incidence of complications, the operation time, the amount of surgical bleeding, hospitalization time, postoperative bone graft fusion rate, and follow-up time, were collated and analyzed. 


\section{Data extraction}

Two researchers used a unified Microsoft Excel (Microsoft, the United States) spreadsheet to independently conduct literature screening and data extraction. Any disagreements were resolved through discussion. The main extracted data included the following: (I) basic information related to the publication such as title, name of the first author, published journal, and year of publication; (II) basic characteristics of the patients including gender, age, and number of cases; (III) specific operation of intervention measures and followup time; and (IV) clinical efficacy evaluation indicators including the VAS, the ODI, and treatment score, complication rate, operative time, operative blood loss, hospitalization time, postoperative fusion rate of bone graft, and follow-up time.

\section{Literature quality evaluation and bias risk assessment}

Two researchers repeatedly evaluated the bias risk of the literature included in the study in strict accordance with the evaluation criteria of RCTs. Any disagreements were resolved through discussion. The evaluation criteria included the following: random sequence generation, allocation concealment, blinding of participants and personnel, blinding of outcomes, incomplete outcome data, selective reporting, and other bias. If the included literature fully satisfies the quality evaluation criteria, the risk of bias is low and the literature is considered grade A. If one or more quality evaluation criteria is only partially satisfied, there is likely moderate bias in the literature and it is considered a grade B literature. If one or more quality evaluation criteria are not satisfied at all, the risk of bias in the study is high and it is recorded as a grade $\mathrm{C}$ literature.

\section{Statistical analysis}

Statistical analysis was performed using Stata SE 15.0 software. Odds ratio (OR) was used for counting data, and mean difference (MD) was used for measurement data. The bias risk assessment chart in the Rev Man 5.3 software was used to evaluate the risk bias of the references. After sorting and screening the data, the Rev Man 5.3 software was used to draw the charts. Each effect was expressed by $95 \%$ CI. A $\mathrm{P}$ value $>0.01$ and $\mathrm{I}^{2}<50 \%$ indicated little heterogeneity among the included studies and the fixed effects model was used for meta-analysis. A $\mathrm{P}$ value $<0.01$ and $\mathrm{I}^{2}>50 \%$ indicated large heterogeneity among the included studies and the random effects model was used for meta-analysis.

\section{Sensitivity analysis}

The random effects model and the fixed effects model were used to compare the results, and the reliability of the combined results was analyzed according to the consistency of the data. Funnel plots were used to determine the presence of publication bias.

\section{Results}

\section{Basic characteristics of the included literature}

A total of 85 documents were retrieved from the database searches and 41 articles were obtained by searching the register. There were 21 duplicate publications and 17 unqualified papers which were excluded. After 9 papers were eliminated for other reasons, 79 papers were screened. A total of 13 articles were excluded, including 6 reviews, 4 studies with incomplete data indicators, and 3 studies where the subjects did not have spondylolisthesis. Finally, 6 articles (10-15) were obtained for meta-analysis. The flow chart in Figure 1 shows the literature retrieval process. The quality evaluation showed that 3 articles (50\%) were classified as grade A, 2 articles $(33.33 \%)$ were grade B, and 1 article $(16.67 \%)$ was grade C.

All 6 included literatures were small sample studies, with sample sizes ranging from 40 to 2,061 cases. There was a total of 2,349 patients. All 6 articles reported the VAS, ODI, and treatment score, incidence of complications, operation time, amount of surgical bleeding, hospitalization time, postoperative bone graft fusion rate, and follow-up time. Table 1 shows the basic characteristics of the included literatures.

\section{Risk bias evaluation}

The risk bias assessment of the included literatures was performed using the Rev Man 5.3 software and Figures 2 and 3 show the evaluation chart and the summary chart, respectively. Among the $6 \mathrm{RCTs}, 3$ articles described allocation concealment in detail, 1 study described the correct random allocation method, and 2 publications did not use blinding methods.

\section{Meta-analysis of intraoperative bleeding}

The amount of intraoperative bleeding was analyzed in 4 


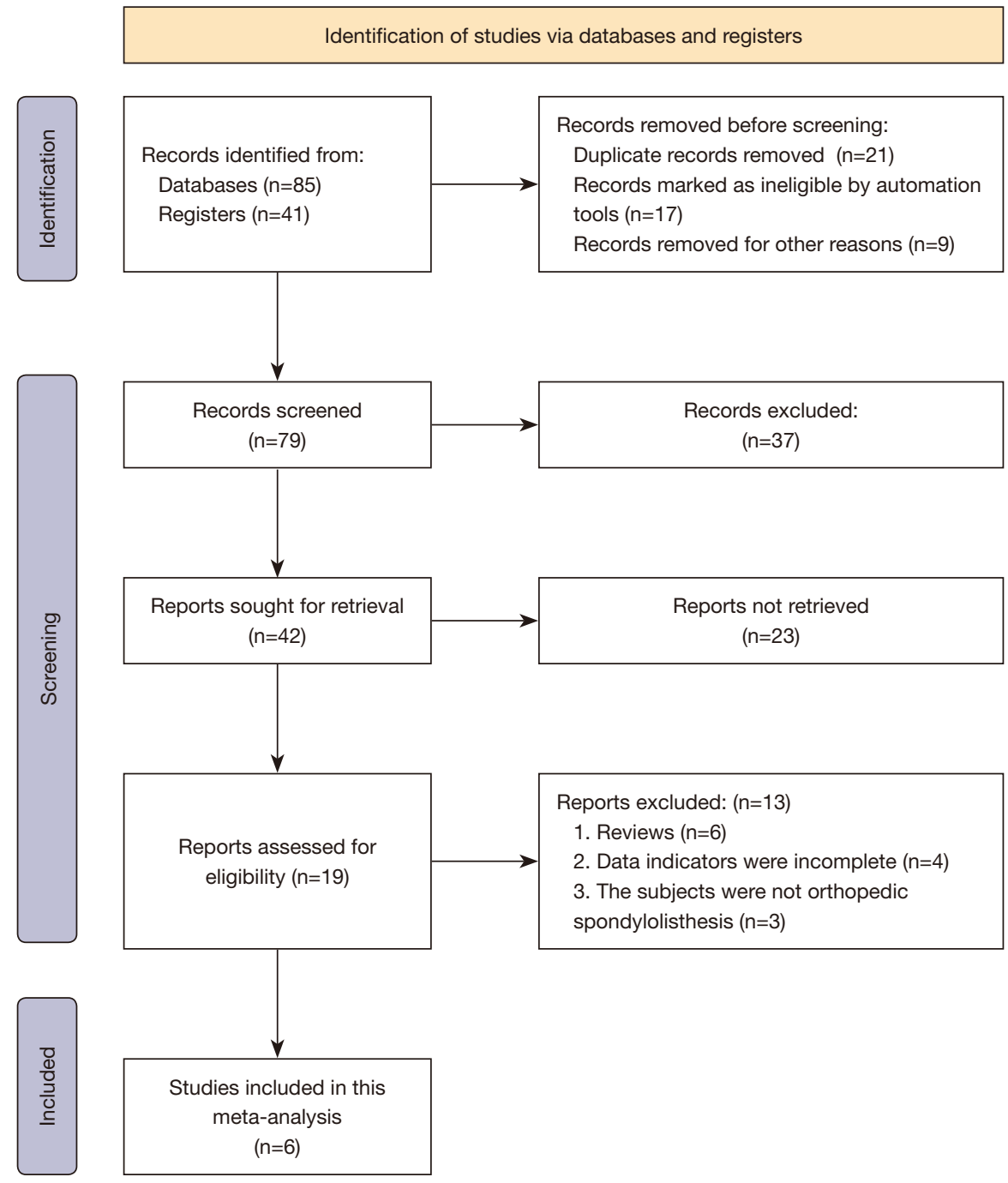

Figure 1 A flow chart showing the literature retrieval process.

Table 1 Basic characteristics of the included literature

\begin{tabular}{lcccccc}
\hline Author & Published year & Cases & Group ALIF (cases) & Group PLF (cases) & Outcome indicators & Follow-up time (ALIF/PLF, month) \\
\hline Kim & 1999 & 40 & 20 & 20 & $\mathrm{f}$ & $3.6 / 2.3$ \\
Lee & 2020 & 53 & 25 & 28 & a, b, c, d, e & $73.6 \pm 2.3 / 54.6 \pm 3.5$ \\
Norheim & 2021 & 2,061 & 570 & 1,491 & $\mathrm{f}$ & - \\
Ohtori & 2011 & 46 & 22 & 24 & a, b, c, d, e & 24 \\
Suk & 2001 & 56 & 21 & 35 & a, b, c, e, f, g & $34.8 / 38.5$ \\
Swan & 2006 & 93 & 47 & 46 & a, b, c, d, e & 24 \\
\hline
\end{tabular}

a, intraoperative blood loss; b, operation time; c, visual analogue scale score; d, Oswestry Disability Index score; e, hospitalization time; f, bone graft fusion rate; g, complications. ALIF, anterior lumbar interbody fusion; PLF, posterolateral fusion. 


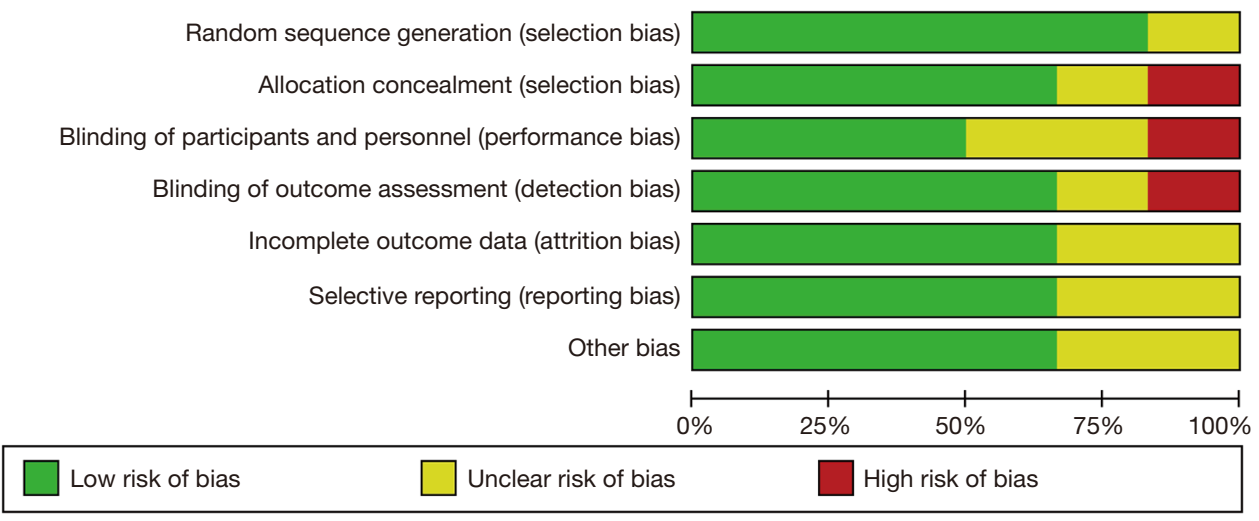

Figure 2 Risk bias evaluation of the included literature.

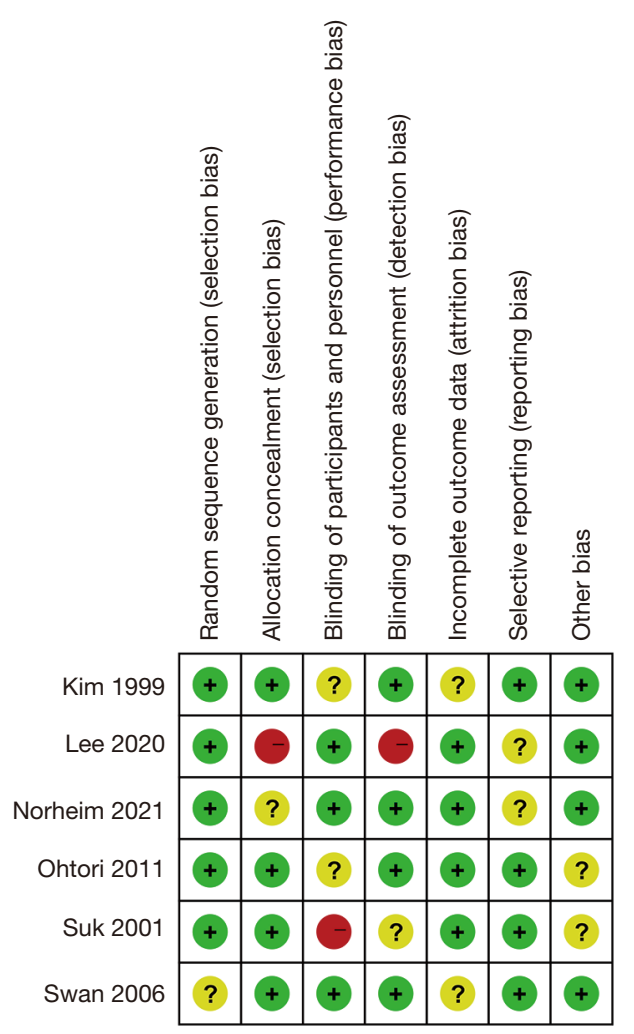

Figure 3 A summary of the risk bias evaluation for the included literature.

RCTs, all of which used continuous variables to describe the data. There was a total of 115 patients in the ALIF group and 133 patients in the PLF group. There was heterogeneity among the studies $\left[\chi^{2}=274.27\right.$; degrees of freedom $\left.(\mathrm{df})=3 ; \mathrm{I}^{2}=99 \%>50 \%\right]$. The horizontal line of the
95\% CI of all studies is to the left of the invalid vertical line. The random effects model showed that intraoperative blood loss was significantly different between patients treated with ALIF and those treated with PLF $[Z=3.34$; mean difference $(\mathrm{MD})=-142.54$; $95 \%$ CI: -226.17 to -58.92 ; $\mathrm{P}=0.0008$; Figure 4]. Funnel chart shows that the circle and midline of the study are basically symmetrical, and one or two documents are deleted arbitrarily, and the combined effect is almost unchanged, which shows that the research accuracy is high and there is no bias in publication (Figure 5).

\section{Meta-analysis of operation time}

A total of 4 literatures reported the operation time using continuous variables, including 115 ALIF cases and 133 PLF cases. There was heterogeneity among the studies $\left(\chi^{2}=21.73 ; \mathrm{df}=3 ; \mathrm{I}^{2}=86 \%>50 \% ; \mathrm{P}<0.0001\right)$ and the random effects model revealed that there was a significant difference in the operation time between the ALIF group and the PLF group ( $\mathrm{Z}=5.45 ; \mathrm{MD}=-54.31 ; 95 \% \mathrm{CI}:-73.83$ to -34.79 ; $\mathrm{P}<0.00001$; Figure 6). Funnel chart shows that the circle and midline of the study are basically symmetrical, and one or two documents are deleted arbitrarily, and the combined effect is almost unchanged, which shows that the research accuracy is high and there is no bias in publication (Figure 7).

\section{Meta-analysis of the VAS score}

A total of 4 publications reported the VAS score using continuous variables, including 115 patients in the ALIF group and 133 patients in the PLF group. There was heterogeneity among the studies $\left(\chi^{2}=27.10\right.$; $\mathrm{df}=3$; 


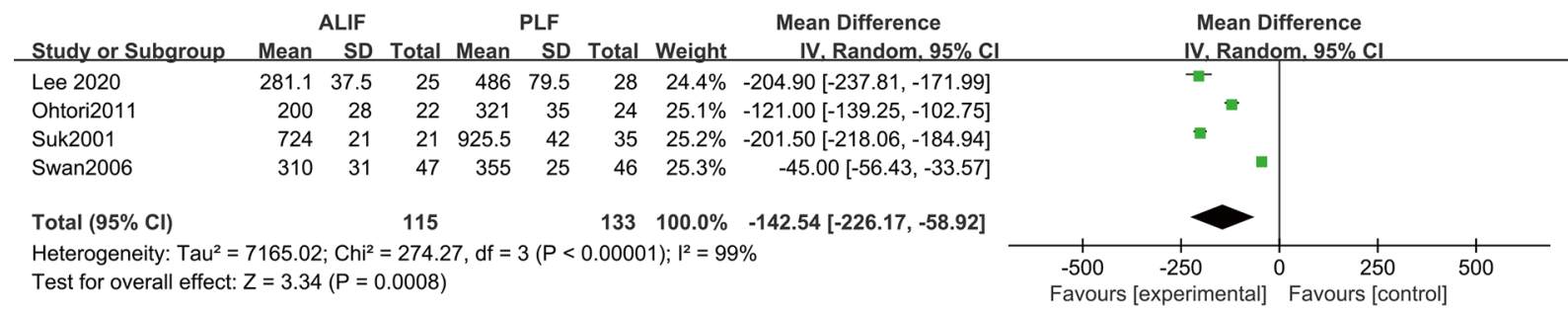

Figure 4 A forest diagram using the random effects model to analyze the amount of intraoperative bleeding.

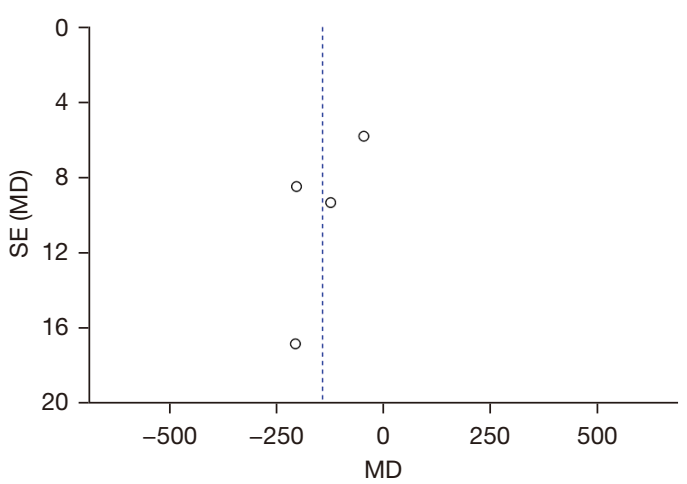

Figure 5 A funnel plot examining the amount of intraoperative bleeding.

$\left.\mathrm{I}^{2}=89 \%>50 \% ; \mathrm{P}<0.00001\right)$ and the random effects model showed that there was significant difference in VAS scores between the ALIF group and the PLF group $(\mathrm{Z}=3.55 ; \mathrm{MD}=-1.04 ; 95 \% \mathrm{CI}:-1.62$ to $-0.47 ; \mathrm{P}=0.0004$; Figure 8). Funnel chart shows that the circle and midline of the study are basically symmetrical, and one or two documents are deleted arbitrarily, and the combined effect is almost unchanged, which shows that the research accuracy is high and there is no bias in publication (Figure 9).

\section{Meta-analysis of ODI score}

The ODI scores were analyzed in 3 literatures, including 94 patients in the ALIF group and 98 patients in the PLF group. There was heterogeneity among the studies $\left(\chi^{2}=11.02 ; \mathrm{df}=2 ; \mathrm{I}^{2}=82 \% ; \mathrm{P}=0.004\right)$ and the random effects model demonstrated that there was significant difference in the ODI score between the ALIF group and the PLF group $(\mathrm{Z}=3.07$; $\mathrm{MD}=-6.33 ; 95 \% \mathrm{CI}:-10.37$ to -2.28 ; $\mathrm{P}=0.002$; Figure 10). Funnel chart shows that the circle and midline of the study are basically symmetrical, and one or two documents are deleted arbitrarily, and the combined effect is almost unchanged, which shows that the research accuracy is high and there is no bias in publication (Figure 11).

\section{Meta-analysis of hospitalization time}

Improvements in the hospitalization time was reported in 3 literatures as dichotomous variables. There was heterogeneity among the study groups $\left(\chi^{2}=61.28\right.$; $\mathrm{df}=2$; $\left.\mathrm{I}^{2}=97 \% ; \mathrm{P}<0.00001\right)$ and the random effects model showed that there was a significant difference in the hospitalization time between the ALIF group and the PLF group $[\mathrm{Z}=2.39$; $\mathrm{MD}=-1.48,95 \%$ CI: -2.70 to $-0.27 ; \mathrm{P}=0.02$; Figure 12]. Funnel chart shows that the circle and midline of the study are basically symmetrical, and one or two documents are deleted arbitrarily, and the combined effect is almost unchanged, which shows that the research accuracy is high and there is no bias in publication (Figure 13).

\section{Meta-analysis of bone graft fusion rate}

A total of 3 literatures analyzed the bone graft fusion rate using binary variables, including 2102 ALIF patients and 625 PLF patients. There was heterogeneity among the publications $\left(\chi^{2}=20.80 ; \mathrm{df}=2 ; \mathrm{I}^{2}=90 \% ; \mathrm{P}<0.0001\right)$ and the random effects model revealed that there was no significant difference in the bone fusion rate between patients in the ALIF group and patients in the PLF group ( $\mathrm{Z}=0.43$; OR $=0.42 ; 95 \%$ CI: 0.01 to $21.82 ; \mathrm{P}=0.66$; Figure 14$)$. Funnel chart shows that the circle and midline of the study are basically symmetrical, and one or two documents are deleted arbitrarily, and the combined effect is almost unchanged, which shows that the research accuracy is high and there is no bias in publication (Figure 15). 


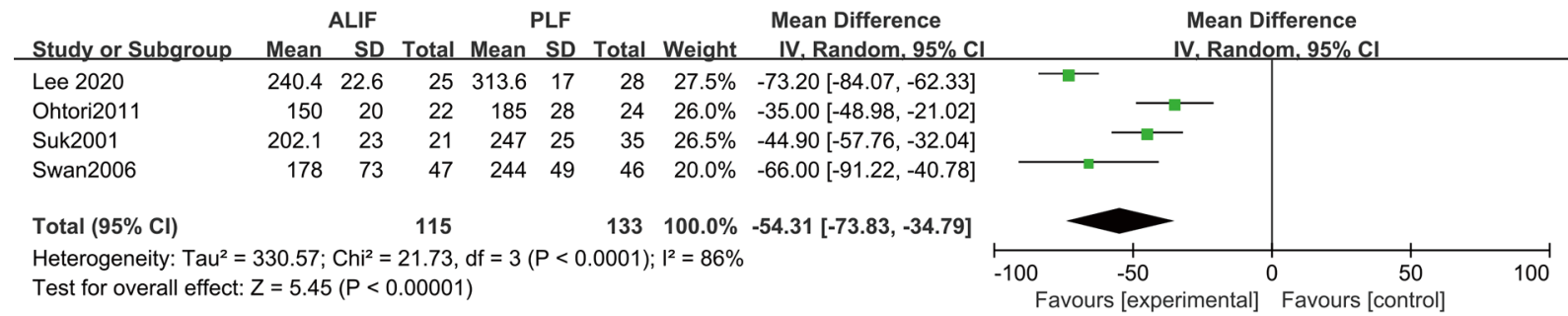

Figure 6 A forest plot using the random effect model to examine operation time.

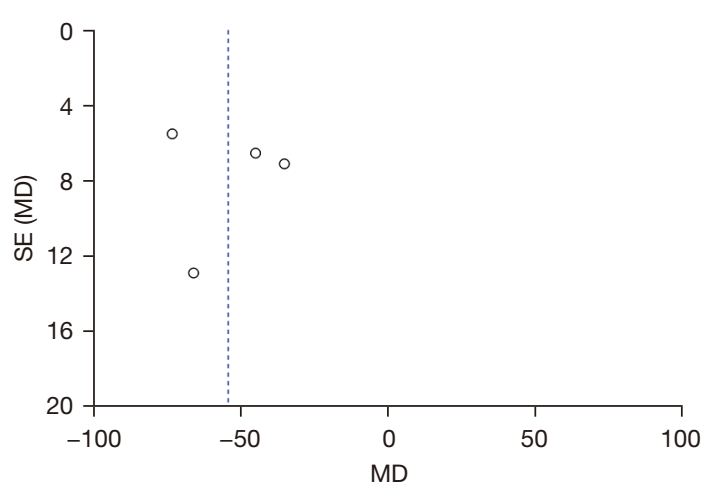

Figure 7 A funnel plot examining operation time.

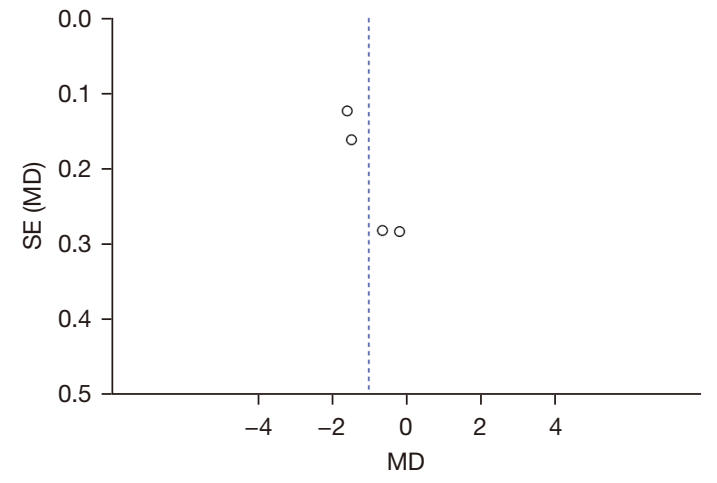

Figure 9 A funnel plot examining VAS score. VAS, visual analogue scale.

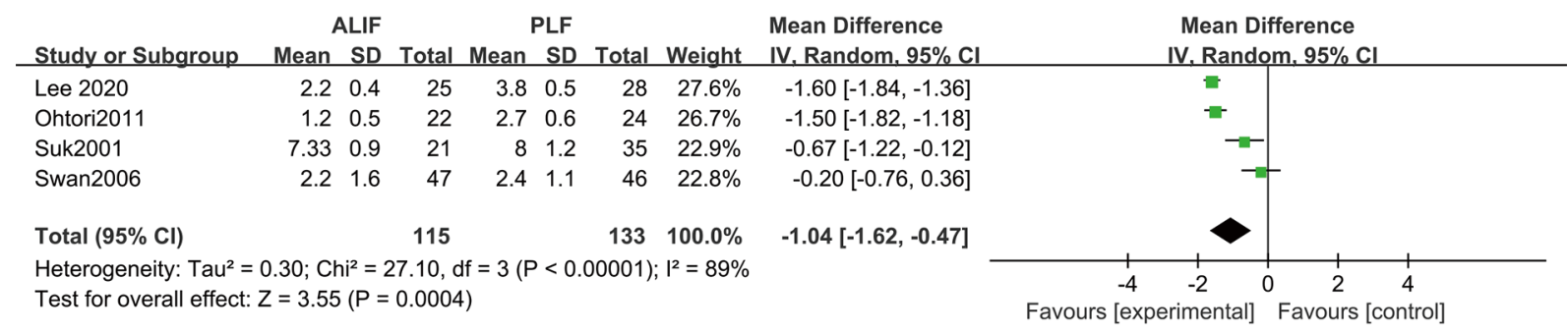

Figure 8 A forest diagram of the VAS score. VAS, visual analogue scale.

\section{Meta-analysis of the rate of complications}

The incidence of complications was analyzed in 2 literatures using binary variable, including 43 cases in the ALIF group and 59 patients in the PLF group. There was no heterogeneity among the publications $\left(\chi^{2}=0.76\right.$; $\left.\mathrm{df}=1 ; \mathrm{I}^{2}=0 \%<50 \% ; \mathrm{P}=0.38\right)$ and the fixed effects model demonstrated that there was no significant difference in the rate of complications between patients who underwent ALIF and patients who underwent PLF $(\mathrm{Z}=0.92 ; \mathrm{OR}=1.64$; 95\% CI: 0.57 to $4.72 ; \mathrm{P}=0.36$; Figure 16 ). Funnel chart shows that the circle and midline of the study are basically symmetrical, and one or two documents are deleted arbitrarily, and the combined effect is almost unchanged, which shows that the research accuracy is high and there is no bias in publication (Figure 17).

\section{Discussion}

Spondylolisthesis is a common spinal disease in which displacement of the vertebral body can increase the tension of the nerve root, cause clinical manifestations such as low back pain and lower limb radiation pain, and greatly reduce 


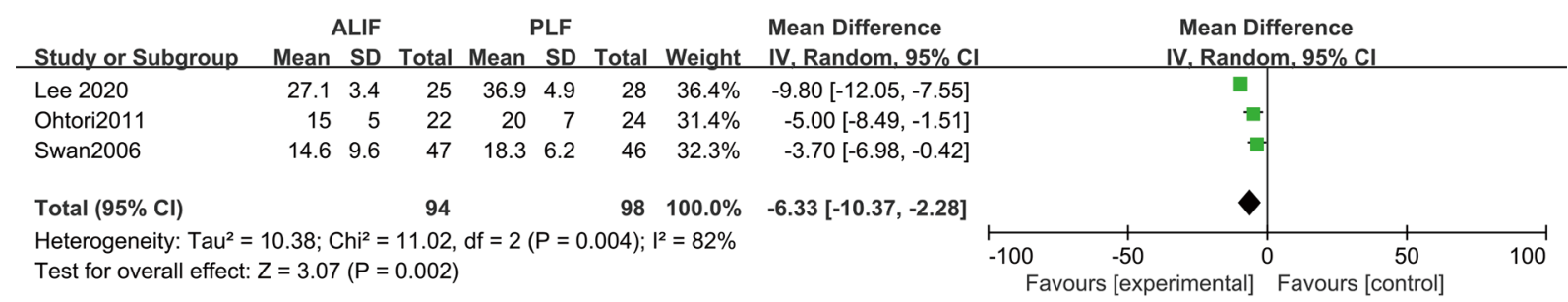

Figure 10 A forest map of the ODI score. ODI, Oswestry Disability Index.

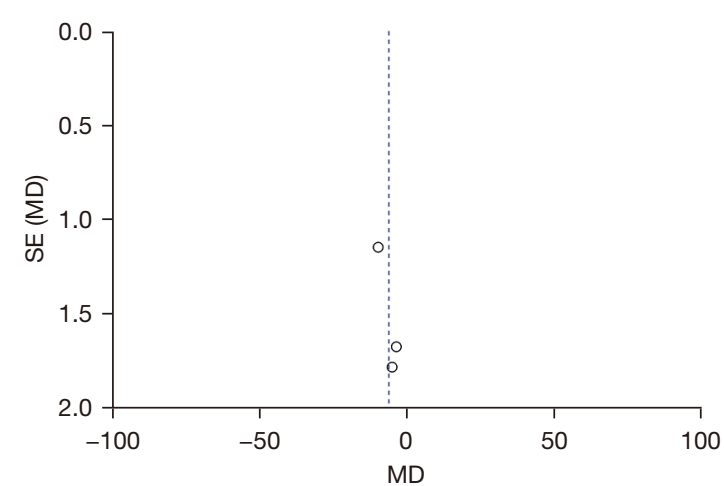

Figure 11 A funnel plot examining ODI score. ODI, Oswestry Disability Index.

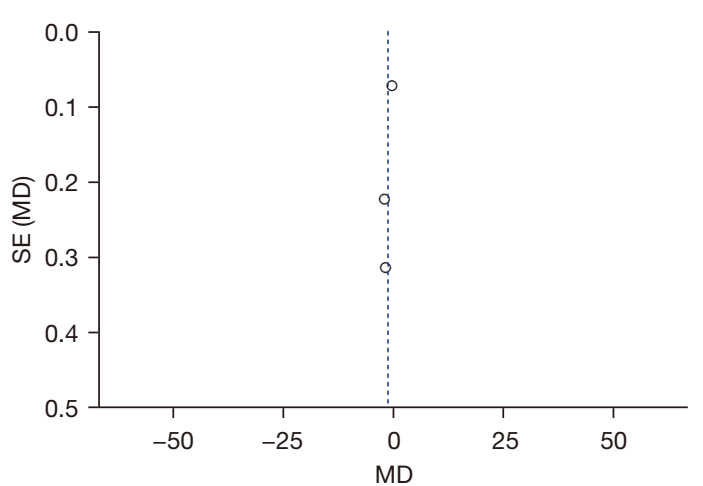

Figure $13 \mathrm{~A}$ funnel plot of the hospitalization time.

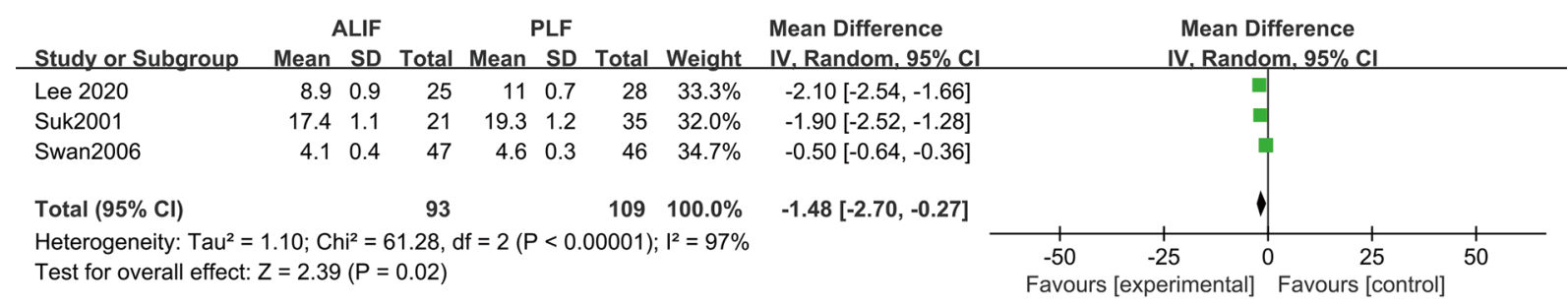

Figure 12 A forest map of the hospitalization time.

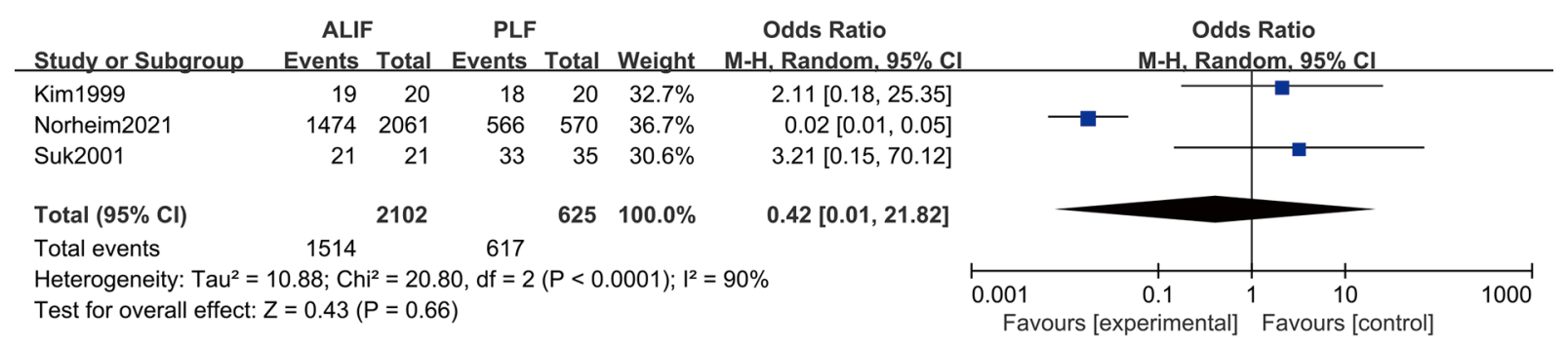

Figure $14 \mathrm{~A}$ forest plot analyzing the bone graft fusion rate. 


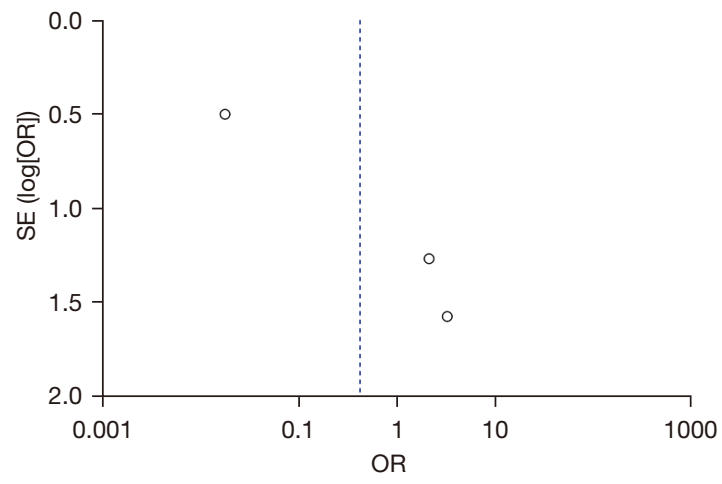

Figure 15 A funnel plot of the bone graft fusion rate.

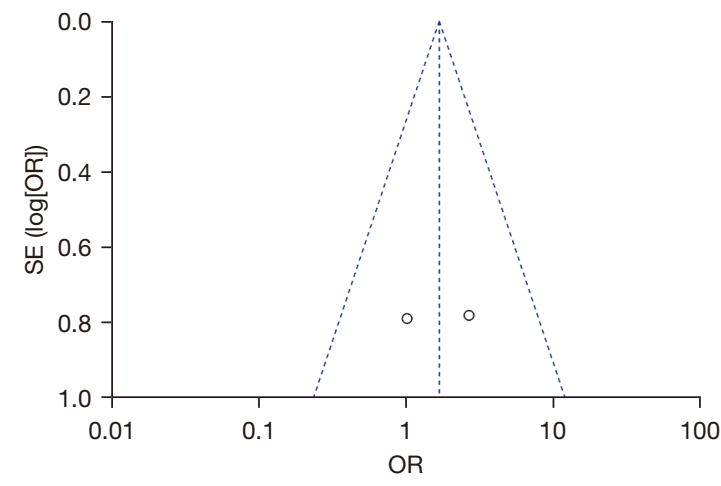

Figure 17 A funnel plot of the incidence of complications.

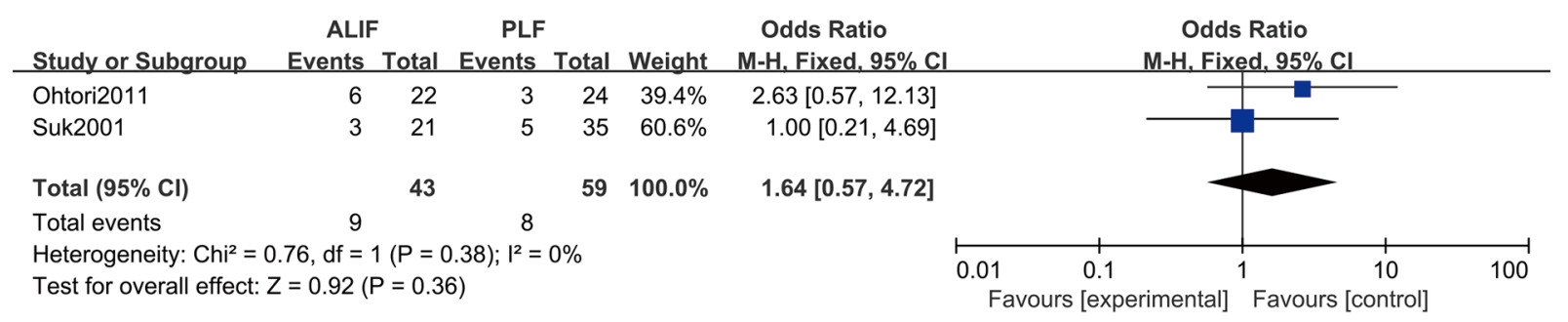

Figure 16 A forest map showing the incidence of complications.

the quality of life of patients (16-18). Surgical treatment restores the anatomical structure of the spine by fusing the spondylolisthesis vertebral body with the adjacent vertebral body, relieves the compression on the nerves, and improves the clinical symptoms of patients by reconstructing the stability of the spine (19).

In recent years, with the development of spinal internal fixation technology, ALIF has contributed significantly to the treatment of lumbar spondylolisthesis (20-22). This study conducted a meta-analysis on outcome indicators including the VAS, the ODI score, time of operation, and fusion rate. The analysis of intraoperative blood loss showed that there was significant difference between ALIF group and PLF group, and the blood loss of ALIF group was lower than that of PLF group. This shows that ALIF can reduce the exposure of vascular muscle tissue, and the amount of blood loss of nerve and blood vessels damaged by removing font bone and exposing transverse bone is less, and the ALIF group has obvious advantages. Compared with PLF group, the operation time of ALIF group was statistically significant. ALIF group did not need to cut the posterior longitudinal ligament of the patient's spine, which reduced the difficulty of surrounding anatomical relationship and took less time (23). Therefore, the operation time of ALIF is shorter than that of PLF, which is consistent with the research results of Spiker et al. [2019] (24). There was no significant difference in VAS score and ODI score between ALIF group and PLF group. The VAS scores of patients with two fusion methods decreased significantly, even if there was no statistical significance, but it also showed that the two fusion methods achieved good clinical effect in improving patients' pain and function. There was significant difference between ALIF group and PLF group in the fusion rate of bone graft. ALIF can directly check the reduction quality of spondylolisthesis through the posterior median approach under direct vision, bite off the lamina, ligamentum flavum and part of the posterior column structure during decompression, and can use less force to reduce the spine (25-27). PLF did not interfere with the intervertebral disc and other structures during the operation, and the reduction effect of spondylolisthesis was not good (28). In addition, both ALIF and PLF surgical methods are combined with internal fixation and bone grafting for spinal fusion, which can obtain satisfactory spinal stability and satisfy patients' routine early functional exercise. 
The quality evaluation showed that 3 articles (50\%) were classified as grade A, 2 articles (33.33\%) were grade $\mathrm{B}$, and 1 article (16.67\%) was grade $\mathrm{C}$, indicating that the high quality of the study is sufficient, the quality of the included experiments is good, and the reliability is high. The funnel charts showed that the circles representing the included studies are basically symmetrical with the midline, indicating that there is no publication bias, and the final conclusion is reliable.

\section{Conclusions}

This meta-analysis explored the clinical efficacy of ALIF compared to PLF in the treatment of spondylolisthesis. The results confirmed that ALIF can effectively improve the degree of spondylolisthesis, provide superior structural stability, and ensure surgical effect. This study was limited by the small number of included literatures, and future high-quality, large sample, randomized controlled experiments are warranted to verify these results. Moreover, the indicators included in the literatures were not comprehensive. For example, the lack of relevant data regarding comorbidities and years of illness may lead to a certain bias in the results. In conclusion, this study is a supplement to previous studies and has significance in guiding the clinical treatment of patients with spondylolisthesis.

\section{Acknowledgments}

Funding: None.

\section{Footnote}

Reporting Checklist: The authors have completed the PRISMA reporting checklist. Available at https://dx.doi. org/10.21037/apm-21-3330

Conflicts of Interest: All authors have completed the ICMJE uniform disclosure form (available at https://dx.doi. org/10.21037/apm-21-3330). The authors have no conflicts of interest to declare.

Ethical Statement: The authors are accountable for all aspects of the work in ensuring that questions related to the accuracy or integrity of any part of the work are appropriately investigated and resolved.
Open Access Statement: This is an Open Access article distributed in accordance with the Creative Commons Attribution-NonCommercial-NoDerivs 4.0 International License (CC BY-NC-ND 4.0), which permits the noncommercial replication and distribution of the article with the strict proviso that no changes or edits are made and the original work is properly cited (including links to both the formal publication through the relevant DOI and the license). See: https://creativecommons.org/licenses/by-nc$\mathrm{nd} / 4.0 \%$.

\section{References}

1. Goodnough LH, Koltsov J, Wang T, et al. Decreased estimated blood loss in lateral trans-psoas versus anterior approach to lumbar interbody fusion for degenerative spondylolisthesis. J Spine Surg 2019;5:185-93.

2. Liu XY, Wang YP, Qiu GX, et al. Meta-analysis of circumferential fusion versus posterolateral fusion in lumbar spondylolisthesis. J Spinal Disord Tech 2014;27:E282-93.

3. Thalgott JS, Sasso RC, Cotler HB, et al. Adult spondylolisthesis treated with posterolateral lumbar fusion and pedicular instrumentation with AO DC plates. J Spinal Disord 1997;10:204-8.

4. Jazini E, Gum JL, Glassman SD, et al. Cost-effectiveness of circumferential fusion for lumbar spondylolisthesis: propensity-matched comparison of transforaminal lumbar interbody fusion with anterior-posterior fusion. Spine J 2018;18:1969-73.

5. Kerolus M, Turel MK, Tan L, et al. Stand-alone anterior lumbar interbody fusion: indications, techniques, surgical outcomes and complications. Expert Rev Med Devices 2016;13:1127-36.

6. Chandra V, Singh RK. Anterior lumbar inter-body fusion with instrumentation compared with posterolateral fusion for low grade isthmic-spondylolisthesis. Acta Orthop Belg 2016;82:23-30.

7. Galimberti F, Lubelski D, Healy AT, et al. A Systematic Review of Lumbar Fusion Rates With and Without the Use of rhBMP-2. Spine (Phila Pa 1976) 2015;40:1132-9.

8. Juricek M, Rehak L, Horvath J, et al. Quality of life after elective lumbar spinal fusions. Bratisl Lek Listy 2010;111:290-5.

9. Vamvanij V, Fredrickson BE, Thorpe JM, et al. Surgical treatment of internal disc disruption: an outcome study of four fusion techniques. J Spinal Disord 1998;11:375-82 .

10. Kim NH, Lee JW. Anterior interbody fusion versus 
posterolateral fusion with transpedicular fixation for isthmic spondylolisthesis in adults. A comparison of clinical results. Spine (Phila Pa 1976) 1999;24:812-6; discussion 817.

11. Lee DG, Park CK, Lee DC. Clinical and radiological comparison of 2 level anterior lumbar interbody fusion with posterolateral fusion and percutaneous pedicle screw in elderly patients with osteoporosis. Medicine (Baltimore) 2020;99:e19205.

12. Norheim EP, Royse KE, Brara HS, et al. PLF+PS or $\mathrm{ALIF}+\mathrm{PS}$ : which has a lower operative nonunion rate? Analysis of a cohort of 2,061 patients from a National Spine Registry. Spine J 2021;21:1118-25.

13. Ohtori S, Koshi T, Yamashita M, et al. Singlelevel instrumented posterolateral fusion versus noninstrumented anterior interbody fusion for lumbar spondylolisthesis: a prospective study with a 2-year followup. J Orthop Sci 2011;16:352-8.

14. Suk KS, Jeon CH, Park MS, et al. Comparison between posterolateral fusion with pedicle screw fixation and anterior interbody fusion with pedicle screw fixation in adult spondylolytic spondylolisthesis. Yonsei Med J 2001;42:316-23.

15. Swan J, Hurwitz E, Malek F, et al. Surgical treatment for unstable low-grade isthmic spondylolisthesis in adults: a prospective controlled study of posterior instrumented fusion compared with combined anterior-posterior fusion. Spine J 2006;6:606-14.

16. Mobbs RJ, Phan K, Malham G, et al. Lumbar interbody fusion: techniques, indications and comparison of interbody fusion options including PLIF, TLIF, MI-TLIF, OLIF/ATP, LLIF and ALIF. J Spine Surg 2015;1:2-18.

17. Ahlquist S, Park HY, Gatto J, et al. Does approach matter? A comparative radiographic analysis of spinopelvic parameters in single-level lumbar fusion. Spine J 2018;18:1999-2008.

18. Safaee MM, Tenorio A, Osorio JA, et al. The effect of anterior lumbar interbody fusion staging order on perioperative complications in circumferential lumbar fusions performed within the same hospital admission. Neurosurg Focus 2020;49:E6.

Cite this article as: $\mathrm{Xu} \mathrm{X}, \mathrm{Li} \mathrm{X}$, Yang T. A systematic review and meta-analysis of the clinical efficacy of anterior lumbar interbody fusion in the treatment of orthopedic spondylolisthesis. Ann Palliat Med 2021;10(12):12607-12617. doi: 10.21037/apm-21-3330
19. Afathi M, Zairi F, Devos P, et al. Anterior lumbar sagittal alignment after anterior or lateral interbody fusion. Orthop Traumatol Surg Res 2017;103:1245-50.

20. Campbell RC, Mobbs RJ, Lu VM, et al. Posterolateral Fusion Versus Interbody Fusion for Degenerative Spondylolisthesis: Systematic Review and Meta-Analysis. Global Spine J 2017;7:482-90.

21. Caprariu R, Popa I, Oprea M, et al. Reduction of spondylolisthesis and sagittal balance correction by anterior lumbar interbody fusion (ALIF). Int Orthop 2021;45:997-1001.

22. Ghogawala Z, Dziura J, Butler WE, et al. Laminectomy plus Fusion versus Laminectomy Alone for Lumbar Spondylolisthesis. N Engl J Med 2016;374:1424-34.

23. Uçar BY, Özcan Ç, Polat Ö, et al. Transforaminal Lumbar Interbody Fusion For Lumbar Degenerative Disease: Patient Selection And Perspectives. Orthop Res Rev 2019;11:183-9.

24. Spiker WR, Goz V, Brodke DS. Lumbar Interbody Fusions for Degenerative Spondylolisthesis: Review of Techniques, Indications, and Outcomes. Global Spine J 2019;9:77-84.

25. Varshneya K, Medress ZA, Jensen M, et al. Trends in Anterior Lumbar Interbody Fusion in the United States: A MarketScan Study From 2007 to 2014. Clin Spine Surg 2020;33:E226-30.

26. Proietti L, Perna A, Ricciardi L, et al. Radiological evaluation of fusion patterns after lateral lumbar interbody fusion: institutional case series. Radiol Med 2021;126:250-7.

27. Tan LA, Rivera J, Tan XA, et al. Clinical and Radiographic Outcomes After Minimally Invasive Transforaminal Lumbar Interbody Fusion-Early Experience Using a Biplanar Expandable Cage for Lumbar Spondylolisthesis. Int J Spine Surg 2020;14:S39-44.

28. Cho JY, Goh TS, Son SM, et al. Comparison of Anterior Approach and Posterior Approach to Instrumented Interbody Fusion for Spondylolisthesis: A Meta-analysis. World Neurosurg 2019;129:e286-93.

(English Language Editor: J. Teoh) 